\title{
Evaluation of the Intermittent Preventive Therapy in Pregnancy using Sulphadoxine/ Pyrimethamine (IPTp-SP) Program in Chiredzi District, Zimbabwe, 2021
}

\author{
Amanda Sarah Thakataka \\ University of Zimbabwe \\ Joseph Mberikunashe \\ Ministry of Health and Child Care \\ More Mungati \\ Elizabeth Glaser Pediatric AIDS Foundation
}

\section{Tsitsi Patience Juru}

University of Zimbabwe

Addmore Chadambuka ( $\nabla$ achadambuka1@yahoo.co.uk)

University of Zimbabwe College of Health Sciences https://orcid.org/0000-0003-2407-1172

Notion Tafara Gombe

African Field Epidemiology Network

\section{Mufuta Tshimanga}

University of Zimbabwe Faculty of Medicine: University of Zimbabwe College of Health Sciences

\section{Research}

Keywords: Malaria, Intermittent Preventive Treatment in Pregnancy, Sulphadoxine/ Pyrimethamine, Zimbabwe

Posted Date: November 9th, 2021

DOI: https://doi.org/10.21203/rs.3.rs-1016538/v1

License: (9) This work is licensed under a Creative Commons Attribution 4.0 International License. Read Full License 


\section{Abstract}

Background Intermittent Preventive Treatment in Pregnancy (IPTp) with Sulphadoxine/ Pyrimethamine $(\mathrm{SP})$ is used for the prevention of malaria among pregnant women in Zimbabwe. The program is integrated into routine Antenatal care (ANC) where a minimum of three doses is recommended during each pregnancy. The third dose coverage for Chiredzi District has consistently been below the $80 \%$ target coverage. We evaluated IPTp implementation in Chiredzi to understand the reasons for underperformance.

Methods An analytical cross-sectional study was conducted using a Process-Outcome evaluation with the Logical Framework Approach. We interviewed 50 women in postnatal care and determined ANC services accessed using their ANC booklets. Health workers were interviewed for knowledge of the program. Stock availability and drug delivery records were reviewed using checklists at five high-volume sites. Epi Info 7.2.4.0 ${ }^{\text {TM }}$ was used for data capturing and analysis. The software was used to calculate frequencies, medians, proportions, odds ratios and p-values at a $95 \%$ confidence interval. The outcome variable was receiving $\geq 3$ doses of SP which was computed against the independent variables such as client-related characteristics to determine which factors were contributory using univariate and bivariate analysis.

Results Only 12/50 (24\%) women received $\geq 3$ doses of SP during their pregnancy. Two (4\%) women started their ANC visits in the first trimester. Thirty-three (66\%) had missed at least one SP dose during their ANC visit because of medicine unavailability. Knowing the number of times SP is given (OR 11.9; $95 \% \mathrm{Cl} 2.54-55.8$ ) and having attended at least 4 ANC visits (OR 13.6; 95\% $\mathrm{Cl} 1.59-116.0$ ) increased the likelihood of receiving adequate SP dosing.

Conclusions The IPTp-SP program in Chiredzi District was underperforming, we attributed this largely to health system factors. Erratic supply and stock-outs of SP resulted in clients missing SP doses. Clients also missed SP doses because of late ANC booking and lack of knowledge on IPTp benefits. Improving stock management and raising community awareness on prevention of malaria among pregnant women was recommended.

\section{Background}

Malaria in pregnancy (MiP) is a noteworthy public health problem, with considerable risks to the mother and her foetus or neonate. In areas of low Plasmodium falciparum transmission, where levels of acquired immunity are low, women are highly susceptible to episodes of severe malaria, which can result in intrauterine deaths, premature delivery, or spontaneous abortion or the death of the mother. In high transmission zones, pregnant women will have asymptomatic infections. This results in maternal anaemia and placental parasitaemia leading to low birth weight and associated poor quality of life for the new-born and increased risk of perinatal mortality [1]. 
$\mathrm{MiP}$ is estimated to account for at least $10 \%$ of all low birth weight among new-born babies resulting in 70000 to 200000 infant deaths each year worldwide. At least 35\% of all pregnancies in 2019 were exposed to malaria, increasing the risk of these adverse outcomes [1, 2]. Masvingo Province has both high and low malaria burden areas, with Chiredzi District having the highest burden. In 2020 the malaria incidence in Chiredzi District was 49 per 1,000 population at risk. At least $25 \%(65,000)$ of the population are women of childbearing age (15-49 years) at risk of MiP [3].

Using Intermittent Preventive Treatment in Pregnancy (IPTp) as a malaria preventive measure has proven beneficial for both the pregnant woman and her unborn child. It is now widely adopted in malariaendemic areas worldwide [1]. To complement this prevention method, the World Health Organisation (WHO) recommends the delivery and use of insecticide-treated nets with prompt and effective case management. In moderate to high transmission areas, administration of IPTp monthly from the second trimester is recommended. Three doses of Sulphadoxine/ Pyrimethamine (SP) are adequate dosing and should ideally be administered as directly observed therapy in antenatal care [4]. These recommendations are supported by findings from a study by Kayentao et al. in 2013 comparing 2 and 3 doses of IPTp-SP where at least three doses were associated with higher birth weight and lesser risk of low birth weight [5]. IPTp coverages of over $80 \%$ have been postulated to avert almost 20000 low birth weights amongst newborn children in Sub-Saharan Africa [1, 2].

The Zimbabwe IPTp program targets women attending ANC in the high perennial and high seasonal transmission strata. IPTp-SP is implemented in an integrated approach with the ANC service delivery package in line with the current WHO protocols. Chiredzi District is one of the 26 districts implementing IPTp-SP in Zimbabwe with baseline IPTp3 coverage of $52.8 \%$ against a target of $80 \%$. Despite the program being resourced with staff training and stock availability, the district has been consistently underachieving. The 2016 WHO ANC model recommended early antenatal care booking of pregnancies and a minimum of 8 ANC visits for each pregnancy, giving the health care provider more contact with the pregnant woman and thus provides more opportunities for SP administration to eligible pregnant women [4]. As a result, IPTp coverages are expected to have improved or to be seen to be improving. We, therefore, evaluated IPTp implementation in Chiredzi to understand reasons for underperformance using a Logical Framework Approach [2]. Figure 1.

\section{Methods}

\section{Study Design}

An analytical cross-sectional study was conducted using a process-outcome evaluation with a Logical Framework Approach.

\section{Study Setting}

Chiredzi District is one of the seven districts in Masvingo Province with a projected population of 413207 in the year 2020 [6]. Heavy rainfall patterns contribute to the high incidence of malaria in the district. 
There is heavy reliance on tea and sugar cane farming. Chiredzi District is among the top 10 districts with the highest burden in the Zimbabwe. The district has 38 public health facilities classified as 4 hospitals (1 district hospital, 1 mission hospital and 2 private hospitals) and 34 clinics.

\section{Study Population}

Postnatal women being followed up in postnatal care within 6 weeks post-delivery were enrolled into the study. To get more information on service delivery we selected health care providers in the Family and Child Health departments as participants. The district health managers served as key informants assisting in providing information on policies and triangulating information from other participants. The ANC cards and booklets for the postnatal women were reviewed to assess the services provided at ANC bookings and during follow-up visits. We reviewed stock availability and drug delivery records.

\section{Sample Size Determination}

A minimum sample size of 50 postnatal women was calculated using the Dobson formula: $n=Z_{a}{ }^{2}(p)(1-$ p) $/$ delta ${ }^{2}$, assuming $Z_{a}=1.96$, delta is 0.05 , a confidence interval is $95 \%$, non-response rate is $10 \%$, based on a study by Holtz et al., 2004 where the 2 nd dose coverage of IPT-SP was 39.7\%; $p=0.03$ [7]. Using the same formula and assumptions, a minimum sample size of 20 health workers was calculated based on a study by Bello and Oni in Nigeria where the offering antenatal obstetric care for less than 5 years was associated with being more knowledgeable on the WHO IPTp-SP recommendations, p=0.012 [8].

\section{Sampling and participants}

We purposefully selected five high-volume health facilities, accounting for $73 \%$ (5385 of 7375 deliveries in 2020 (DHIS)), of the district's deliveries. These included four hospitals serving as referral centres for all the facilities in the district and a polyclinic serving the urban population. Key informants totalling ten IPTp district level program managers were purposefully selected. These were the District Medical Officer (DMO), District Nursing Officer (DNO), District Pharmacist, Hospital Matrons (2), and in-charge nurses in the $\mathrm{FCH}$ departments (5). Using the number of deliveries in 2020 for the selected health facilities we proportionally sampled the number of health workers and postnatal women to be enrolled at each facility. We purposefully enrolled health workers in the $\mathrm{FCH}$ departments and randomly selected postnatal women from those attending postnatal care. This was done until we reached the desired sample size per facility.

\section{Data Collection Methods and Tools}

Health workers were interviewed using an interviewer-administered questionnaire in English. The tool targeted health workers who implement the program activities. Prior scheduling of the interview was done to avoid services delivery interruption. The information collected was on their demographic characteristics, their qualifications, training on IPTp, IPTp services they offered, and assessment of knowledge on IPTp and guidelines. Key informants were interviewed using a key informant guide, to validate the data collected from the primary interviewees. We also used an observation checklist to validate the responses of the informants. A structured interviewer-administered questionnaire in local language was used to collect information from women in postnatal care. We collected information on 
their sociodemographic, IPTp experience and knowledge on IPTp-SP during pregnancy. We verified the information concerning ANC services from the ANC cards and booklets.

\section{Data Analysis}

Electronic questionnaires were created in Epi Info 7.2.4.0 ${ }^{\mathrm{TM}}$ (CDC, 2020). Data were entered into these questionnaires, cleaned and verified. The same software package was used to calculate frequencies, medians, proportions, odds ratios and p-values. All calculations were done at a $95 \%$ confidence interval. Microsoft Excel 365 was used to draw tables and graphs. The outcome variable was receiving $\geq 3$ doses or SP which was computed against the independent variables, the client-related characteristics to determine contributing factors using univariate and multivariate analysis.

Results

\section{Characteristics of study participants}

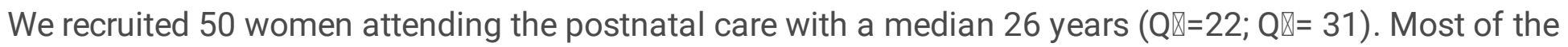

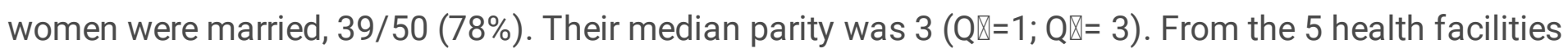
visited we recruited 20 health workers, of these 17(85\%) were females. Nine (45\%) were midwives, 7(35\%) were registered general nurses and $4(20 \%)$ were primary care nurses. The median years in service was 8 years $(\mathrm{Q} \backslash=3.5 ; \mathrm{Q} \mathbb{Q}=14)$.

\section{Performance of the IPTp program in Chiredzi}

\section{Inputs of the IPTp program}

All the health facilities had updated policy guidelines, charts and ANC registers. One facility had the older version of ANC guidelines also displayed which recommended a minimum of 4 ANC visits per pregnancy, IPTp-SP to be initiated at 16 weeks or quickening and 2 doses were considered adequate. Most women, $40 / 50(80 \%)$ had ANC booklets, while the remainder had improvised exercise books.

Only two women had booked their first ANC visit in the first trimester, the majority $30 / 50$ (60\%) booked in the 2 nd trimester and the rest $18 / 50$ (36\%) booked in the 3rd trimester. Four months of SP stock was considered adequate stock. Out of five, three health facilities were stocked out of SP, one was understocked with stocks lasting less than 4 months and one was overstocked with months of stock above 7 months. Thirty-three women (66\%) had missed at least one SP dose during their ANC visit because of medicine unavailability. Table 1.

\section{Processes involved in running the IPTp program}

The last didactic health worker training on MiP was in 2018, thus all health workers recruited after 2018 had not been trained. Out of $20,11(55 \%)$ health workers had ever been trained. The district health managers visit all the health facilities, and these visits are integrated with other public health programs, however, records showed that only two out of the recommended four malaria mentorship visits were done 
in 2020 per health facility in the district. All the five health facilities had a service point for the administration of SP under direct observation, but it was not currently in use at two health facilities, to avoid sharing of cups during the Covid-19 pandemic.

Half of all women had received at least one session of health education on malaria during pregnancy. Only $28 / 50$ (56\%) of the postnatal women had attended at least 4 ANC visits, and of these only $11 / 28(39 \%)$ had received at least 3 doses of SP. None had attained the recommended 8 ANC visits. Table 1.

\section{Outputs and Outcomes of the IPTp program}

All the health facilities visited were offering IPTp services. Out of the 43 women who had received at least one dose of SP, only 29 (67\%) had received via DOT. Only 12/50 (24\%) of the participants had three or more doses of IPTp-SP during their pregnancy as recommended by WHO, 30/50 (60\%) had 2 doses and the majority $43 / 50(86 \%)$ had received the 1 st dose. Table 1 . Four women were diagnosed and treated for malaria during pregnancy. Of these two had attended at least 4 ANC visits, three had missed at least one dose of SP in the ANC and two had received one SP dose, one received 2 doses and the 4th woman had received 5 doses. These factors were not statistically significant with $p>0.05$. 
Table 1

Performance of Intermittent Preventive Treatment in Pregnancy indicators in Chiredzi District, 2021 Indicator Target Actual Comment Inputs

Number of health facilities with policy guidelines and charts

Number of health facilities ANC Registers

Postnatal women with ANC Cards

ANC attendance in the 1 st trimester

Number of health facilities reporting stockout of SP in ANC

Number of women who missed SP dose because of stock out
5

5

50

50

4

4

5

40

$(80 \%)$

$2(4 \%) \quad$ Inadequate

03 Inadequate

$0 \quad 33 / 50 \quad$ Inadequate $(66 \%)$

\section{Processes}

Percentage of ANC staff trained in the control of MiP

Malaria-focused mentorship and support visits

Number of health facilities administering SP under direct observation

Number of women receiving health education on MiP

The percentage of pregnant women attending at least four ANC visits

Proportion of women who attended at least 4ANC visits who received at least 3 doses of SP

\section{Outputs}

Number of health facilities offering IPTp

Number of women who received IPTp-SP dose under direct observation

\section{Outcomes}

Proportion of eligible women who received IPTp1

Proportion of eligible women who received IPTP2

Proportion of eligible women who received IPTp3
$20 \quad 11(55 \%) \quad$ Inadequate

42 Inadequate

$53 \quad$ Inadequate

$50 \quad 25(50 \%) \quad$ Inadequate

$50 \quad 28 \quad$ Inadequate (56\%)

28

11(39\%) Inadequate

5

5

Adequate

43

29

(67\%)

Inadequate

43

Adequate

(86\%)

$50 \quad 30(60 \% \quad$ Inadequate

50
12

(24\%)

\section{Knowledge}




\section{Clients' knowledge of Malaria in Pregnancy}

Thirty-three women (66\%) knew that headache and fever were the major symptoms of malaria. According to 37 women $(74 \%)$ one may develop malaria after a bite from an infected mosquito and $34 / 50(68 \%)$ knew that the use of an insecticide-treated net or IRS could prevent this. Forty-one (82\%) knew that a pregnant woman could get malaria, while only $28(56 \%)$ knew that the consequences of malaria in pregnancy included severe disease to the pregnant woman, a risk of LBW infant, miscarriage and even maternal mortality. Regarding IPTp use, 30/50 (60\%) knew that IPTp is a drug used to prevent malaria during pregnancy, but only $11 / 50(22 \%)$ and $6 / 50(12 \%)$ knew how many times a pregnant woman was supposed to get IPTp and its benefits to the mother and her unborn child, respectively. Figure 2.

\section{Health worker knowledge of the IPTp program}

Most of the health workers knew that SP was the medicine recommended for prevention of malaria in pregnancy. They also knew that SP is administered under direct observation 18/20 (90\%) and the correct intervals between SP administration 19/20 (95\%). Sixteen (80\%) stated there was a benefit in using IPTp, however, just 10 (50\%) mentioned the benefit of reduced maternal and infant mortality and three mentioned the reduction in maternal anaemia, prematurity, and LBW. Only one health worker correctly mentioned the gestational age at which the first dose of SP should be administered whilst eight (40\%) knew the correct number of minimum doses recommended per pregnancy. Figure 3.

\section{Client-related factors associated with receiving at least 3 doses of SP in pregnancy}

There were no differences in the likelihood of receiving at least 3 doses by age groups, marital status, parity, level of education, employment status, mode of travel or having received health education during pregnancy. However, women living in urban areas, with knowledge on the minimum number of SP doses they were meant to receive and having attended at least 4 ANC visits had an increased likelihood of receiving adequate SP doses whilst being of the apostolic religious sector was a hindrance to receiving adequate doses of SP. These factors were statistically significant. Table 2. 
Table 2

Client-related factors associated with receiving at least 3 doses of Sulphadoxine/ Pyrimethamine in Pregnancy

\begin{tabular}{|c|c|c|c|c|c|}
\hline Variable & & $\begin{array}{l}\geq 3 \mathrm{SP} \\
\text { doses }\end{array}$ & $\begin{array}{l}<3 \text { SP } \\
\text { doses }\end{array}$ & OR $(95 \% \mathrm{Cl})$ & $\begin{array}{l}\mathrm{p}- \\
\text { value }\end{array}$ \\
\hline \multirow[t]{3}{*}{ Age group } & $<25$ years & 4 & 20 & Ref* & - \\
\hline & $26-30$ years & 4 & 8 & $\begin{array}{l}0.40(0.08- \\
2.00)\end{array}$ & 0.26 \\
\hline & $>31$ years & 4 & 10 & $\begin{array}{l}0.50(0.10- \\
2.43)\end{array}$ & 0.39 \\
\hline \multirow[t]{2}{*}{ Marital Status } & Married & 9 & 30 & \multirow{2}{*}{$\begin{array}{l}0.80(0.17- \\
3.66)\end{array}$} & \multirow[t]{2}{*}{0.77} \\
\hline & Not married & 3 & 8 & & \\
\hline \multirow[t]{2}{*}{ Gravidity } & Primigravid & 2 & 11 & \multirow{2}{*}{$\begin{array}{l}0.49(0.09- \\
2.61)\end{array}$} & \multirow[t]{2}{*}{0.40} \\
\hline & Multigravid & 10 & 27 & & \\
\hline \multirow[t]{2}{*}{ Level of education } & $\begin{array}{l}\text { Secondary and } \\
\text { above }\end{array}$ & 8 & 22 & \multirow[t]{2}{*}{$\begin{array}{l}1.45(0.37- \\
5.68)\end{array}$} & \multirow[t]{2}{*}{0.59} \\
\hline & $\begin{array}{l}\text { Primary or } \\
\text { none }\end{array}$ & 4 & 16 & & \\
\hline \multirow[t]{2}{*}{ Religion } & Apostolic & 2 & 20 & \multirow{2}{*}{$\begin{array}{l}0.18(0.03- \\
0.93)\end{array}$} & \multirow[t]{2}{*}{0.03} \\
\hline & Other religion & 10 & 18 & & \\
\hline \multirow[t]{2}{*}{ Occupation } & Employed & 13 & 7 & \multirow{2}{*}{$\begin{array}{l}2.79(0.86- \\
9.01)\end{array}$} & \multirow[t]{2}{*}{0.08} \\
\hline & Unemployed & 12 & 18 & & \\
\hline \multirow[t]{2}{*}{ Number of ANC visits } & $\geq 4$ & 11 & 17 & \multirow{2}{*}{$\begin{array}{l}13.6(1.59 \\
-116.0)\end{array}$} & \multirow[t]{2}{*}{0.004} \\
\hline & $<4$ & 1 & 21 & & \\
\hline \multirow[t]{2}{*}{ Place of residence } & Urban & 9 & 16 & \multirow{2}{*}{$\begin{array}{l}4.13(0.96- \\
17.7)\end{array}$} & \multirow[t]{2}{*}{0.047} \\
\hline & Rural & 3 & 22 & & \\
\hline \multirow{2}{*}{$\begin{array}{l}\text { Mode of travel to the nearest } \\
\text { health facility }\end{array}$} & Walk & 10 & 27 & \multirow{2}{*}{$\begin{array}{l}2.03(0.38- \\
10.8)\end{array}$} & \multirow[t]{2}{*}{0.40} \\
\hline & Drive/vehicle & 2 & 11 & & \\
\hline \multirow{2}{*}{$\begin{array}{l}\text { Receiving Health Education in } \\
\text { ANC }\end{array}$} & Yes & 8 & 17 & \multirow{2}{*}{$\begin{array}{l}2.47(0.63- \\
9.63)\end{array}$} & \multirow[t]{2}{*}{0.19} \\
\hline & No & 4 & 21 & & \\
\hline \multirow[t]{2}{*}{ Knowledge of IPTp and its use } & Correct & 19 & 11 & \multirow{2}{*}{$\begin{array}{l}2.59(0.81- \\
8.29)\end{array}$} & \multirow[t]{2}{*}{0.10} \\
\hline & Incorrect & 8 & 12 & & \\
\hline
\end{tabular}




\begin{tabular}{|c|c|c|c|c|c|}
\hline Variable & & $\begin{array}{l}\geq 3 \text { SP } \\
\text { doses }\end{array}$ & $\begin{array}{l}<3 \text { SP } \\
\text { doses }\end{array}$ & OR (95\% Cl) & $\begin{array}{l}\mathrm{p}- \\
\text { value }\end{array}$ \\
\hline \multirow{2}{*}{$\begin{array}{l}\text { Number of times IPTp is given in } \\
\text { each pregnancy }\end{array}$} & Correct & 7 & 4 & \multirow{2}{*}{$\begin{array}{l}11.9(2.54- \\
55.8)\end{array}$} & \multirow[t]{2}{*}{$<0.001$} \\
\hline & Incorrect & 5 & 34 & & \\
\hline
\end{tabular}

\section{Discussion}

We conducted a descriptive cross-sectional study using a Process-Outcome evaluation with the Logical Framework Approach to evaluate the IPTp Program in Chiredzi District and to determine the reasons for the district failing to achieve the target for IPTp-SP coverages. While all five facilities visited were implementing the IPTp program and had adequate policy guidelines and stationery for documentation of ANC services offered, other health system factors contributed to the program failing to meet its targets. These factors were medicine stock-outs, non-adherence to program guidelines, health worker knowledge deficits, inconsistencies in service delivery, inadequate training and mentorship. To a lesser extent, clientrelated factors contributed to the low IPTp coverage in Chiredzi District. These were late ANC bookings, religion, and lack of appreciation of the IPTp program benefits and function.

Our study demonstrated the availability of adequate guideline documents to guide the health workers' approaches at participating health facilities. Only one still displayed outdated guidelines. This is a good starting point to ensure adherence to the expected program protocols. Adequacy of monitoring and evaluation (M\&E) tools enable clinicians to document services provided and subsequently assess the performance of the IPTp programs. Assuming completeness of recording we would have reliable information to understand current performance enabling the development of mitigating activities to improve the program. Thus, we can exclude documentation as a cause of poor performance. A study conducted by Sande et al. 2017 also stated that the incorrect documentation on cards and registers could cause problems in IPTp program evaluation [10].

Erratic medicines supply and shortages affect the effective implementation of the IPTp program. Drug supply inconsistencies contribute to missed opportunities for adequate dosing even with high ANC attendance as has been found by other scholars [11]. Resource availability, particularly SP within the health facility, bears on the proper implementation of the IPTp program. In this current study, medicine stock-outs contributed to low coverages, much more than the effects of late booking and the number of ANC attendances. Evidence in Tanzania and Ghana shows that SP shortages do contribute largely to low IPTp coverages $[9,12]$. If district health managers addressed this challenge the program performance will improve.

For a health program to be implemented the system needs trained and skilled personnel. Although there seemed to be adequate numbers of health workers in the $\mathrm{FCH}$ departments the majority were not trained. The majority who had been previously trained have since moved from the facilities or public health system. The failure of the health system to retain trained health workers as was reported in this study 
could have contributed to knowledge and skill deficits. This affects program implementation. In this study, the most deficits were on the benefits of the program and SP administration with regards to when it should be initiated, and the minimum doses required. Lack of knowledge on the correct timing and spacing of SP, adherence to current guidelines, and the benefits of the program have also been demonstrated in other studies $[9,13,14]$.

Our evaluation revealed that there was a lack of regular supervision visits and health worker mentorship. This could explain some poor practices we noted. For instance, the non-adherence to SP administration guidelines despite health workers knowing the correct practice. When SP is self-administered, there is no way of monitoring to validate the adherence and assessing the impact of the intervention may be difficult. All health facilities need to innovate ways to ensure that directly observed administration of SP is done. Displaying outdated ANC guidelines causes mistakes in the administration of malaria preventive medicines and can be identified (and addressed) by adequate supervision. Other studies show that even with guidelines on IPTp in place, compliance to them remains a challenge that needs solving $[4,15,16]$. In another study, it was shown that a year after IPTp was introduced still more women missed their first dose of SP compared with tetanus immunization, even though both interventions were offered at the same time [14]. This goes to highlight the continuous need for health worker sensitization on the program.

Client-related factors also contributed to low IPTp coverages in Chiredzi District. Late ANC booking as was evident affects health services delivery including IPTp. Additionally, drug supply inconsistencies further contributed to missed opportunities for adequate dosing even with high ANC attendance as has been found by other scholars [11]. With most women presenting late for their first ANC visit, opportunities to receive the minimum IPTp package are reduced. This results in reduced protection of pregnant women, and their unborn babies, from malaria. Evidence shows that subsequent doses of SP decline along the IPTp cascade. Hence, starting ANC early offers more opportunities for the completion of the standard IPTp course [7, 13, 17-19]. Contrary to this finding a study in Malawi reported that even in the setting of early ANC booking IPTp coverage may still be suboptimal [7]. Suggesting that other factors can influence IPTp uptake.

In this evaluation, living in an urban area increased the likelihood of receiving the minimum recommended SP doses whilst being of the Apostolic religious sect was a hindrance. These findings are similar to what has been found in other local studies $[18,20]$. Urban residence may mean that access to health care is easier though in this study either having to walk or need a means of transportation to attend ANC did not have significant implications on receiving SP doses.

To increase program performance the beneficiaries of a program should have an adequate understanding of its function and appreciate its benefits. To increase awareness of a health program like the IPTp program, health education imparted from health workers to ANC clients is crucial and increases clients' demand for the intervention. Health workers knowledge deficits could explain the lack of malaria-specific 
education for pregnant women as was evident in this evaluation. Health workers who are knowledgeable of a program may easily pass on the same knowledge to their clients $[11,13,17]$.

In this study, women had good knowledge of malaria transmission and prevention, but deficits were seen in the knowledge of the benefits of IPTp and the dosages. Similarly, other scholars have attributed poor uptake of SP to a lack of knowledge of the IPTp program, its benefits and those of early pregnancy booking $[9,11,12]$. The resulting low SP coverages during pregnancy increases the risk of malaria complications for the pregnant woman and the unborn child $[8,9,17]$. However, another local study seems to suggest otherwise, as no direct relationship between client knowledge on IPTp and utilization of the program intervention was proven [20]. In support of this, some scholars have suggested that client uptake of IPTp by women in low-income countries is a result of poor quality ANC package, inaccessible service due to high cost of getting to the service area, distance to the health facility, and the actual waiting time for the services at the facilities $[7,17,21]$. In this evaluation means of getting to a health facility were not contributory.

Though subjective, as no records were found, less than a 10th of postnatal women reported having been diagnosed and treated for malaria during pregnancy. This was much lower than the prevalence reported by Arnaldo et al., 2018 in southern Mozambique [21]. Whilst we cannot attribute this to the IPTp-SP program, this finding could signify that other malaria preventive measures were being implemented in the community. Something that can be supported by the good knowledge on malaria prevention by postnatal women.

\section{Limitations}

Our findings may not be generalizable to all health facilities since we only selected high-volume health facilities. We interviewed women who had given birth and were attending the postnatal clinics, and this could have introduced recall bias. Our sample size may be too small to make inferences on the factors associated with receiving at least 3 doses of SP, however useful information that can help hypothesis generation for future studies was obtained from these findings.

\section{Conclusion}

In our evaluation of the IPTp-SP program in Chiredzi District, we found that the program targets were not being reached. The reasons for this are many. Health system factors contributed greatly to low SP coverages and the two factors most evident in our study were erratic supply and stock-outs of SP and deficits in health worker knowledge, skills and practices. The beneficiaries of the program also contributed to inefficiency in program implementation. Women booked ANC late which translated to lesser opportunities for SP administration, and they lacked appreciation of the program and its benefits causing less demand for SP.

\section{Recommendations}


We shared our findings with the health managers and made recommendations. The district needs to improve on stock management of SP, through ensuring the availability of medicines at all health facilities and redistribution of excess stock. Regular supervision and mentorship at all health facilities is needed to address the anomalies that occur and reinforce adherence to guidelines and standard operating procedures. Finally, to address client related, the continued need for community sensitization on early pregnancy booking and malaria prevention in pregnancy was emphasized. This can be achieved through collaborating with community health workers in continuously providing health education community activities and liaising with traditional leaders, educating them, and seeking their support in ensuring male partners provide the necessary support to their pregnant spouses and register pregnancies early.

\section{Public Health Actions}

Erratic supplies of SP were the main reason for poor performance. After sharing the findings with the key stakeholders at the district, province and the National Malaria Control Program, we made efforts to determine stock availability at other Nat Pharm branches. We contacted the Provincial Logistic manager for Nat Pharm and identified that regionally SP was available and could be supplied and distributed to the district. The district pharmacist was tasked to arrange a special order from the regional Nat Pharm branch to the district pharmacy to cushion the shortages from the Zimbabwe Assisted Pull System delivery. The excess stock at Colin Saunders Hospital was taken to the district hospital for redistribution. We ensured the removal of all outdated guidelines and IEC material in the health facilities, in collaboration with the community health nursing department. We highlighted the changes in the updated guidelines to the health workers in the facilities we visited.

\section{Abbreviations}

ANC

Antenatal Care

DHE

District Health Executive

DMO

District Medical Officer

DNO

District Nursing Officer

$\mathrm{FCH}$

Family and Child Health

HSO

Health Studies Office

IPTp

intermittent preventive treatment in pregnancy

IRS

indoor residual spraying 
ITN

insecticide-treated nets

MiP

Malaria in pregnancy

Nat Pharm

National Pharmaceuticals

NMCP

National Malaria Control Program

PMD

Provincial Medical Director

SP

Sulphadoxine/ Pyrimethamine

WHO

World Health Organisation

\section{Declarations}

\section{Ethical approval and consent to participate}

Permission to carry out the study was obtained from the District Medical Officer (DMO) of Chiredzi District, the Provincial Medical Director (PMD), and the Health Studies Office (HSO). Informed written consent was got from study participants and confidentiality was assured, anonymity and privacy were maintained. A detailed explanation of the study was given to all study participants for informed decisionmaking. Participants were told that participation is voluntary, and they could withdraw from the study during the study. Covid-19 infection prevention and control regulations were observed, through keeping interview time to a maximum of 15 mins, maintaining physical distancing, wearing masks by both interviewer and respondent and hand hygiene.

\section{Consent for publication}

Not applicable

\section{Availability of data and materials}

While restrictions to data exist, the data is available for use and sharing.

\section{Competing interests}

The authors declare that they have no competing interests.

\section{Funding}

No external funding was provided for the study. 


\section{Authors' contributions}

AST, JM, MM, TPJ, AC, NTG, and MT: conception, design, and acquisition. AST, JM, MM data analysis and interpretation of data. AST, JM, MM, AC, TPJ wrote the first draft of the manuscript. All authors read and approved the final manuscript for publication.

\section{Acknowledgements}

Special thanks to Provincial Medical Directorate Masvingo Province, District Medical office Chiredzi district, the National Malaria Control Program and the Health Studies Office University of Zimbabwe for the support offered during the entire study. We would also like to extend our sincere gratitude to all the study participants enrolled in the study.

\section{References}

1. World Health Organization. World malaria report 2020: 20 years of global progress and challenges. 2020. https://www.who.int/docs/default-source/malaria/world-malaria-reports/9789240015791double-page-view.pdf?sfvrsn=2c24349d_5. Accessed 13 December 2020.

2. Malaria World. Monitoring and Evaluation of Malaria in Pregnancy Services: Practical Tips and Recommended Indicators. 2020. https://malariaworld.org/blog/malaria-pregnancy-monitoringevaluation-brief. Accessed 4 December 2020.

3. ZimStat POB. ZIMBABWE POPULATION CENSUS 2012. 2012;152.

4. World Health Organization. WHO recommendations on antenatal care for a positive pregnancy experience. 2016 https://www.who.int/publications-detail-redirect/9789241549912. Accessed 19 January 2021.

5. Kayentao K, Garner P, van Eijk AM, Naidoo I, Roper C, Mulokozi A, et al. Intermittent Preventive Therapy for Malaria During Pregnancy Using 2 vs 3 or More Doses of Sulfadoxine-Pyrimethamine and Risk of Low Birth Weight in Africa. JAMA. 2013;309(6):594-604.

6. Zimbabwe National Statistics Agency (ZIMSTAT). Masvingo Province District Population Projections Report (2012 to 2032). 2020;74.

7. Holtz TH, Kachur SP, Roberts JM, Marum LH, Mkandala C, Chizani N, et al. Use of antenatal care services and intermittent preventive treatment for malaria among pregnant women in Blantyre District, Malawi. Trop Med Int Health TM IH. 2004;9(1):77-82.

8. Bello 00, Oni O. Health Workers' Awareness and Knowledge of Current Recommendation of Intermittent Preventive Treatment in Pregnancy in South-Western Nigeria. Ethiop J Health Sci. 2020;30(1):125-134.

9. Vandy AO, Peprah NY, Jerela JY, Titiati P, Manu A, Akamah J, et al. Factors influencing adherence to the new intermittent preventive treatment of malaria in pregnancy policy in Keta District of the Volta region, Ghana. BMC Pregnancy Childbirth. 2019;19(1):424. 
10. Sande S, Zimba M, Mberikunashe J, Tangwena A, Chimusoro A. Progress towards malaria elimination in Zimbabwe with special reference to the period 2003-2015. Malar J. 2017;16(1):295.

11. Mchwampaka WM, Tarimo D, Chacky F, Mohamed A, Kishimba R, Samwel A. Factors affecting uptake of $\geq 3$ doses of Sulfadoxine-Pyrimethamine for malaria prevention in pregnancy in selected health facilities, Arusha region, Tanzania. BMC Pregnancy Childbirth. 2019;19(1):440.

12. Exavery A, Mbaruku G, Mbuyita S, Makemba A, Kinyonge IP, Kweka H. Factors affecting uptake of optimal doses of sulphadoxine-pyrimethamine for intermittent preventive treatment of malaria in pregnancy in six districts of Tanzania. Malar J. 2014;13(1):22.

13. Verhoeff FH, Brabin BJ, Chimsuku L, Kazembe P, Russell WB, Broadhead RL. An evaluation of the effects of intermittent sulfadoxine-pyrimethamine treatment in pregnancy on parasite clearance and risk of low birthweight in rural Malawi. Ann Trop Med Parasitol. 1998;92(2):141-150.

14. van Eijk AM, Ayisi JG, ter Kuile FO, Otieno JA, Misore AO, Odondi JO, et al. Effectiveness of intermittent preventive treatment with sulphadoxine-pyrimethamine for control of malaria in pregnancy in western Kenya: a hospital-based study. Trop Med Int Health TM IH. 2004;9(3):351-360.

15. Brabin BJ, Warsame M, Uddenfeldt-Wort U, Dellicour S, Hill J, Gies S. Monitoring and evaluation of malaria in pregnancy - developing a rational basis for control. Malar J. 2008;7(1):S6.

16. Mace KE, Chalwe V, Katalenich BL, Nambozi M, Mubikayi L, Mulele CK, et al. Evaluation of sulphadoxine-pyrimethamine for intermittent preventive treatment of malaria in pregnancy: a retrospective birth outcomes study in Mansa, Zambia. Malar J. 2015;14(1):69.

17. Mushi V, Mbotwa CH, Zacharia A, Ambrose T, Moshi FV. Predictors for the uptake of optimal doses of sulfadoxine-pyrimethamine for intermittent preventive treatment of malaria during pregnancy in Tanzania: further analysis of the data of the 2015-2016 Tanzania demographic and health survey and malaria indicator survey. Malar J. 2021;20(1):75.

18. Chikwasha V, Phiri I, Chimberengwa P, Bangure D, Rusakaniko S. Predictors of IPTp Uptake among Pregnant Women in the 2010-2011 Zimbabwe Demographic and Health Survey. Rockv Md USA ICF Int. 2014;DHS Working Papers No. 112(Zimbabwe Working Papers No. 13).

19. World Health Organization. Malaria in pregnancy: guidelines for measuring key monitoring and evaluation indicators. 2007. Genève (Suisse). World Health Organization.

20. Chituku S. Knowledge of women of child bearing age on the utilisation of intermittent preventive treatment of malaria in pregnancy at Dangamvura and Sakubva health centers, Mutare, Zimbabwe. 2013. https://zdhr.uz.ac.zw/xmlui/handle/123456789/1121. Accessed 4 January 2021.

21. Arnaldo P, Rovira-Vallbona E, Langa JS, Salvador C, Guetens P, Chiheb D, et al. Uptake of intermittent preventive treatment and pregnancy outcomes: health facilities and community surveys in Chókwè district, southern Mozambique. Malar J. 2018;17(1):109.

\section{Figures}




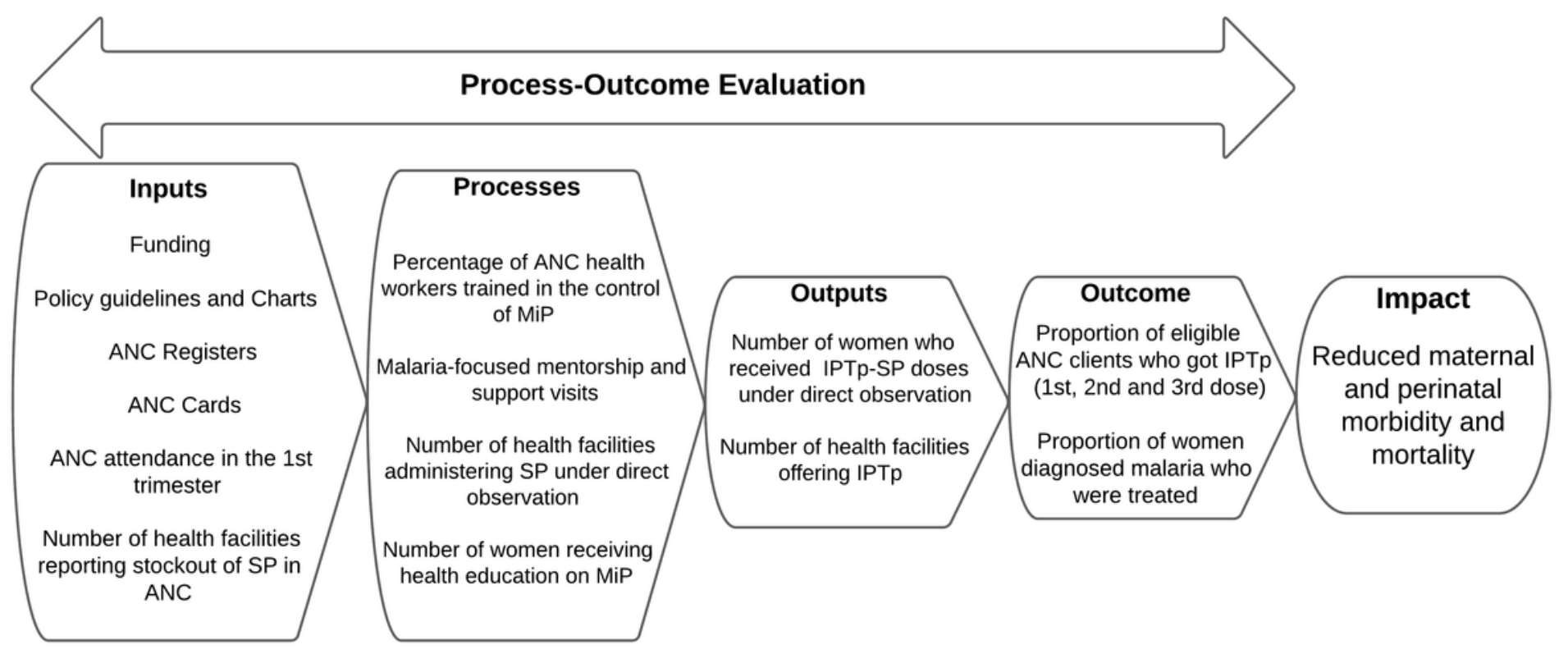

\section{Figure 1}

The Logical Framework Approach. Adopted from Malaria in pregnancy monitoring and evaluation framework Approach [2]

\section{Clients knowledge of Malaria prevention in Pregnancy}

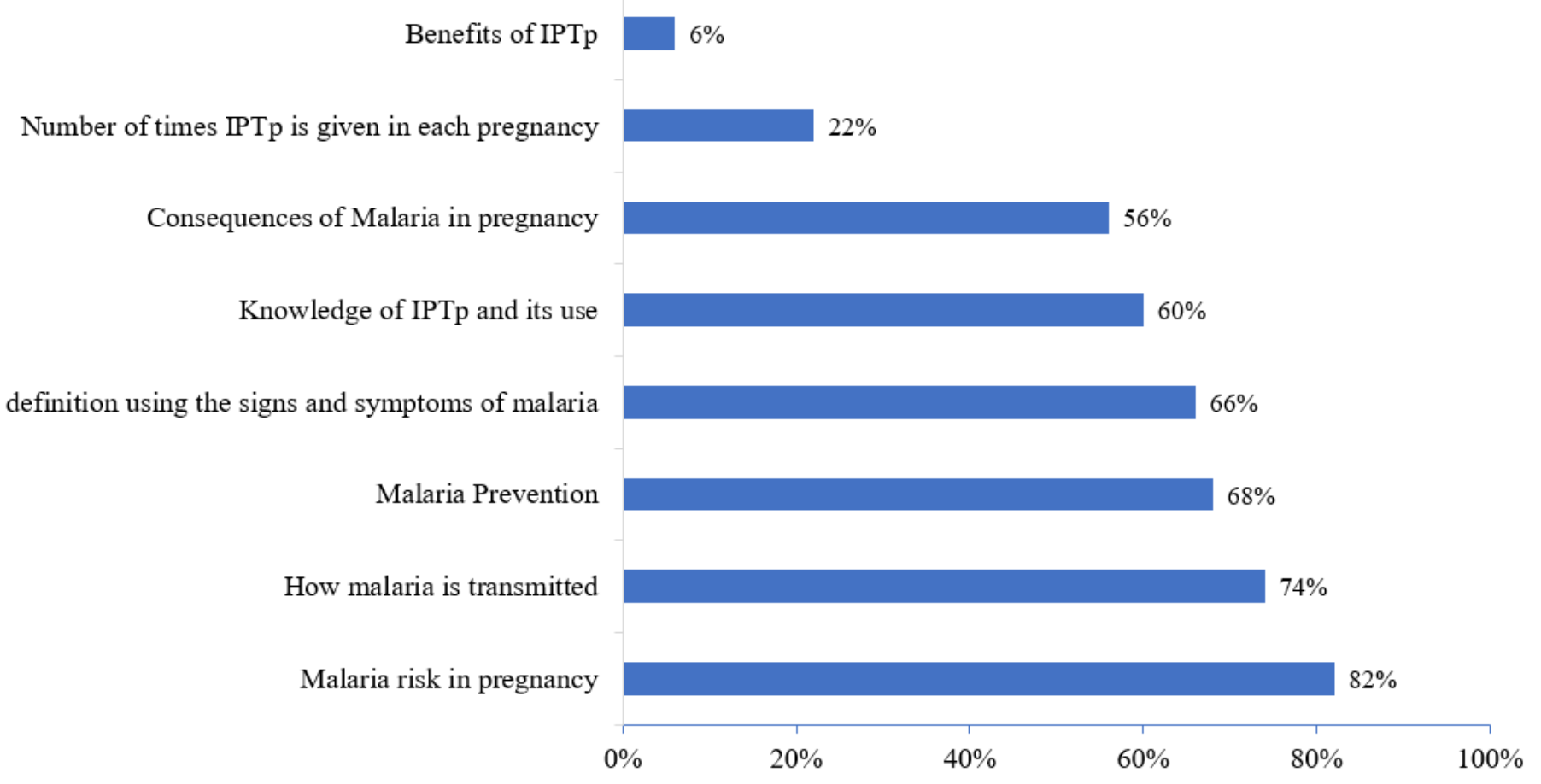

Proportion on postnatal women with knowledge

\section{Figure 2}


Clients' knowledge of Malaria in Pregnancy

Health worker knowledge on IPTp Use

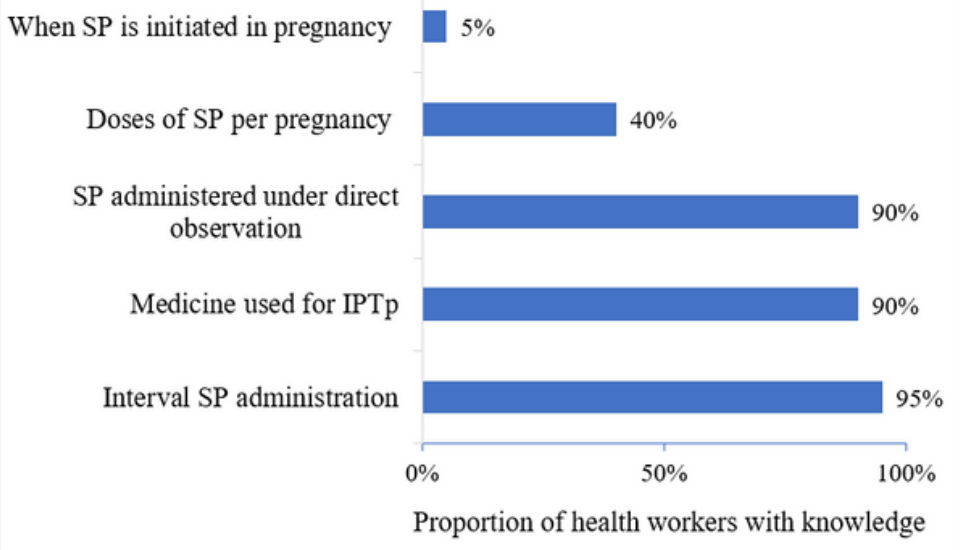

Health worker knowledge on the benefits of IPTp

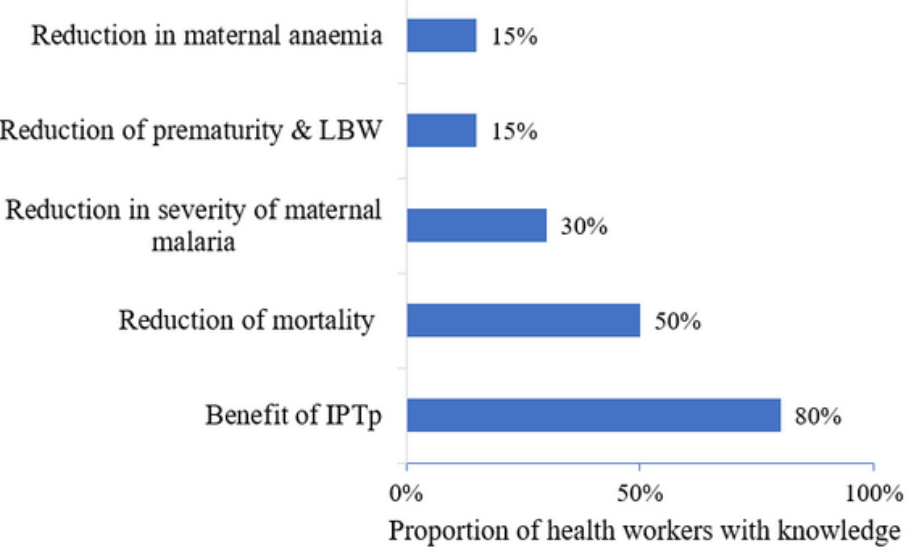

\section{Figure 3}

Health worker knowledge on Intermittent Preventive Treatment in Pregnancy Use and Benefits 